\title{
Nonlinear hole transport through a submicron-size channel
}

\author{
O. Makarovsky, ${ }^{\text {a) }}$ A. Neumann, A. M. Martin, L. Turyanska, A. Patanè, L. Eaves, \\ M. Henini, and P. C. Main \\ School of Physics and Astronomy, University of Nottingham, NG7 2RD United Kingdom \\ S. Thoms and C. D. W. Wilkinson \\ Department of Electronics and Electrical Engineering, University of Glasgow, G12 8LT United Kingdom \\ D. K. Maude and J. C. Portal \\ Grenoble High Magnetic Field Laboratory, MPI-CNRS, F-38042 Grenoble, France
}

(Received 25 September 2002; accepted 13 December 2002)

\begin{abstract}
We investigate hole transport through a submicron-size channel fabricated from a modulation-doped $p$-type GaAs/(AlGa)As single-quantum-well heterostructure. The intense electric field in the channel accelerates the holes beyond the inflection point of the lowest energy subband dispersion curve. This leads to current saturation and negative differential conduction effects in the currentvoltage characteristics. (C) 2003 American Institute of Physics. [DOI: 10.1063/1.1543643]
\end{abstract}

The energy-wave vector, $\varepsilon(k)$, dispersion curves of holes in a quantum well (QW) are highly nonparabolic due to the admixing of the heavy-hole (HH) and light-hole ( $\mathrm{LH})$ subbands by spin-orbit interaction. ${ }^{1,2}$ In particular, the lowest-energy subband (HH1) has an inflection point in $\varepsilon(k)$, corresponding to a maximum hole velocity, phenomenologically similar to that found for Bloch electrons in superlattices. ${ }^{3}$ At energies above this point, the subband has a region with negative effective mass (NEM). It has been proposed that this property can be exploited to develop a class of high-frequency [terahertz $(\mathrm{THz})$ ] generators and detectors in which ballistic holes are accelerated by an intense electric field to their peak velocity in a submicron high-field device. ${ }^{4}$ Alternatively, optical excitation of holes followed by radiative intersubband relaxation could be used as a source of THz radiation. ${ }^{5}$

In this letter, we investigate hot-hole transport through a submicron-size channel fabricated from a modulation-doped p-type GaAs/(AlGa)As single QW heterostructure. Studies of hole transport through narrow constrictions have been reported previously and have shown quantized conductance effects. ${ }^{6}$ Here, we show that holes can be accelerated up to and beyond the inflection point in the highly nonparabolic HH1 subband of the QW, thus leading to current saturation and negative differential conduction effects in the currentvoltage characteristics.

The $\mathrm{GaAs} / \mathrm{Al}_{0.3} \mathrm{Ga}_{0.7} \mathrm{As}$ heterostructure was grown by molecular beam epitaxy on a semi-insulating (SI), (311)Aoriented GaAs substrate. It consists of a $15 \mathrm{~nm}$ wide QW, modulation-doped with $\mathrm{Si}$ acceptors. Transport measurements in standard Hall bars at $T=1.2 \mathrm{~K}$ gave a hole mobility $\mu=50 \mathrm{~m}^{2} / \mathrm{V} \mathrm{s}$ and a sheet density $p=2.1 \times 10^{15} \mathrm{~m}^{-2}$ for the two-dimensional hole gas (2DHG).

Using electron beam lithography, the layer was processed into a four-terminal device in which holes can flow through a narrow constriction between contacts 1 and 3 or 2 and 4 (see Fig. 1). The constriction is formed by dry etching

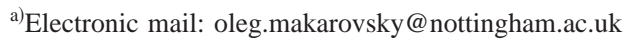

the heterostructure down to the SI substrate. The contact pads allow us to make four-terminal measurements so that the effect of contact resistances can be eliminated. In the current-voltage characteristic, $I(V)$, shown in Fig. 2(a), the voltage $V$ comprises the potential drop across the short and narrow constriction and across the larger low-resistance tapered areas between the constriction and the contact pads. We studied several structures with different geometrical constriction widths, $w$, and/or pitch angles, $\Theta$ (see Fig. 1). Here, we focus on a device with $w=0.5 \mu \mathrm{m}$ and $\Theta=30^{\circ}$. Due to depletion effects, the effective width of the constriction is smaller than $w$. By measuring the conductance of devices with different $w$, we estimate the depletion thickness on each side of the channel to be $\approx 0.1 \mu \mathrm{m}$, giving an effective channel width $w_{\text {eff }} \approx 0.3 \mu \mathrm{m}$.

The low temperature $(T=1.2 \mathrm{~K}) I(V)$ curve of the device presented in Fig. 2(a) is highly nonohmic. Also, it shows two interesting features, $a$ and $b$. These features disappear at $T>10 \mathrm{~K}$ and are absent in $p$-type devices with larger constrictions $(w \geqslant 1 \mu \mathrm{m})$ and in $n$-type devices of similar design. The conductance plot $G(V)=d I / d V$ shown in Fig. 2(a)

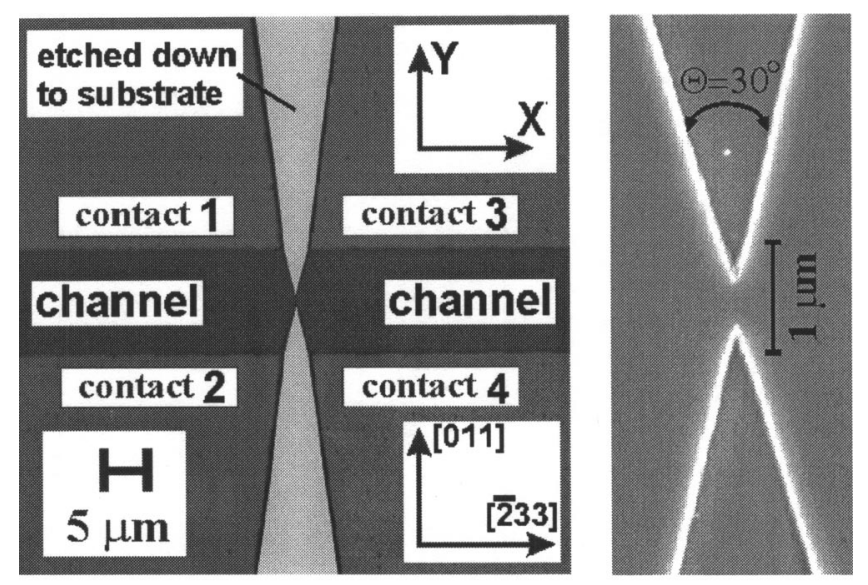

FIG. 1. Left: Optical-microscope image of the device, showing the channel, the constriction, and the contact pads. The channel is directed along the [233] crystallographic direction on the (311)A-oriented GaAs plane. Right: Scanning electron microscope image of the constriction region of the device. 

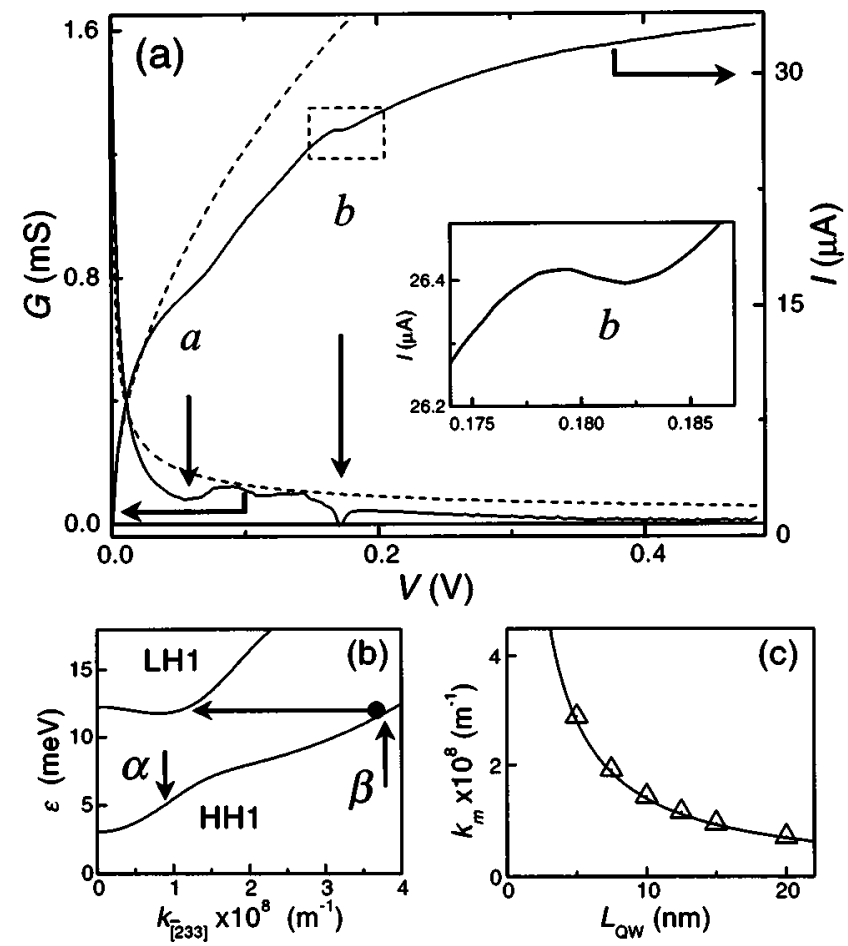

FIG. 2. (a) Current and differential conductance vs applied bias. Dotted lines describe curves $I(V) \sim V^{1 / 2}$ and $G(V) \sim V^{-1 / 2}$. The inset shows feature $b$ in more detail. (b) Energy dispersion curves calculated for a $15 \mathrm{~nm}$ wide $\mathrm{GaAs} /(\mathrm{AlGa}) \mathrm{As} \mathrm{QW}$ grown on a (311)A-oriented GaAs substrate. The arrows indicate features $\alpha$ and $\beta$ in the HH energy dispersion curve that we relate to features $a$ and $b$ in the $G(V)$ plot. (c) $k$ value of the inflection point $\alpha, k_{m}$, as a function of the quantum well width, $L_{\mathrm{QW}}$. The continuous line describes the curve $\gamma L_{\mathrm{QW}}^{-2}$, where $\gamma$ is a constant.

reveals features $a$ and $b$ more clearly. The conductance at $V=0$ has a value of $1.6 \mathrm{mS}$. It is strongly quenched with increasing temperature (e.g., at $T=14 \mathrm{~K}, G$ is reduced by a factor 2) and by the application of a magnetic field, $B_{\perp}$, perpendicular to the plane of the $2 \mathrm{DHG}$ (e.g., at $T=1.2 \mathrm{~K}$, a field of $0.5 \mathrm{~T}$ reduces $G$ by a factor 2 ). The latter effect is probably due to classical magnetoresistance or magneticfield-induced depopulation of one-dimensional states in the channel. At high $B_{\perp}$ and very low bias, quantized Hall effect features corresponding to filling factors 1 and 2 are observed in the conductance in fields of 8.6 and $4.3 \mathrm{~T}$, respectively, indicating that the device processing has not had an adverse effect on the electrical properties.

In order to interpret these data, we first note that, for a parabolic band, a one-dimensional classical ballistic model gives the following $I(V)$ and $G(V)$ characteristics: $I \sim v$ $\sim V^{1 / 2}$ and $G \sim V^{-1 / 2}$, where $v$ is the carrier group velocity. This model provides a crude fit to our data up to $V$ $\approx 50 \mathrm{mV}$. However, the fit is poor at higher bias and the model does not explain features $a$ and $b$. As described later, the data can be understood in terms of holes accelerated by the electric field towards the nonparabolic region of $\varepsilon(k)$. The dispersion curves were numerically calculated for our 15 $\mathrm{nm}$ wide $\mathrm{GaAs} / \mathrm{Al}_{0.3} \mathrm{Ga}_{0.7} \mathrm{As} \mathrm{QW}$ grown on a (311)Aoriented GaAs substrate and are shown in Fig. 2(b). This calculation was performed along the lines of the original work of Luttinger and Kohn ${ }^{7}$ taking into account the anisotropic band parameters for a (311)-oriented QW. ${ }^{8}$ Recent theoretical studies have shown that the distribu- where $\ell_{B}=(\hbar / e B)^{1 / 2}$ is the magnetic length.
Downloaded 23 May 2004 to 128.250 .49 .72 . Redistribution subject to AlP license or copyright, see http://apl.aip.org/ap/ tion of ballistic carriers moving across a narrow and short channel can become unstable in the presence of a NEM region in the carrier energy dispersion curve. At electric fields high enough to accelerate holes toward the first inflection point of the HH1 subband, the $I(V)$ becomes highly nonohmic and high-frequency oscillations in the current are expected at still higher electric fields. ${ }^{4}$ According to this model, the pronounced shoulder in $I(V)$ at $V_{a} \approx 50 \mathrm{mV}$ [feature $a$ in Fig. 2(a)] would arise from holes that are accelerated towards the first inflection point of the HH1 subband, $\alpha$. We also note that at point $a$, the measured value of $I \approx 15 \mu \mathrm{A}$ is quite similar to that $(I \approx 6 \mu \mathrm{A})$ obtained from the relation $I$ $=p e v_{m} w_{\text {eff }}$, where $v_{m}$ is the hole peak group velocity at point $\alpha$ in the calculated dispersion curve shown in Fig. 2(b) $\left(v_{m}=\hbar^{-1} \partial \varepsilon / \partial k=5.8 \times 10^{4} \mathrm{~ms}^{-1}\right.$ at $\left.k_{m}=0.96 \times 10^{8} \mathrm{~m}^{-1}\right)$. However, in our device, a bias of $V_{a} \approx 50 \mathrm{mV}$ is required to accelerate holes up to $\alpha$, whereas in a purely ballistic model, the necessary voltage would be considerably less, $\approx 5 \mathrm{mV}$, corresponding to the energy at $\alpha$. It therefore seems likely that the hole motion through the constriction is at least partially diffusive, i.e., the holes undergo a small number of scattering events before they are accelerated up to $\alpha$ by the electric field. This is consistent with the effective geometric length of our constriction, which we estimate to be $l_{\text {eff }}$ $\approx 0.2 \mu \mathrm{m} .{ }^{9}$ In comparison, previous measurements using a quasiballistic tunnel transistor indicate a path length $l$ $\approx 14 \mathrm{~nm}$ for hot light holes with energies in excess of the energy of the LO phonon, ${ }^{10}$ whereas light holes with lower energies have a longer path length $l \approx 300 \mathrm{~nm} .{ }^{11}$ Also, assuming that at point $a$ in $I(V)$, the mean electric field around the constriction is given by $E_{a} \approx V_{a} / l_{\text {eff }}$, we can use the relation $k_{m}=e E_{a} \tau_{s} / \hbar=0.96 \times 10^{8} \mathrm{~m}^{-1}$ to obtain an estimate for the scattering time, $\tau_{s}$, from our measurements. This gives $\tau_{s} \approx 0.2 \mathrm{ps}$, which is consistent with other values reported in the literature using optical excitation techniques, ${ }^{12,13}$ and a value of $l \approx v_{m} \tau \approx 13 \mathrm{~nm}$.

We can gain further insight into the role of the inflection point at $\alpha$ on the $I(V)$ characteristics by calculating the effect of changing the $\mathrm{QW}$ width, $L_{\mathrm{QW}}$, on the dispersion of the lowest-energy hole subband. As shown in Fig. 2(c), the $k$ value at the inflection point $\alpha, k_{m}$, varies approximately as $1 / L_{\mathrm{QW}}$. This result can be understood qualitatively in terms of the anticrossing of the two lowest-energy subbands as a result of the second order, spin-orbit interaction. ${ }^{1}$ We can mimic the effect of decreasing the well width in a given sample by applying a magnetic field, $B_{\|}$, in the plane of the QW. Such a field has a weak effect on the in-plane motion, but produces an additional quantum confinement along the growth direction, $z$. We use a simple harmonic-oscillator potential, $V_{0}(z)=m \Omega^{2} z^{2} / 2 e$, and its associated Gaussian ground-state eigenfunction to fit our exact calculation of the wave function component $\varphi_{1}(z)$ of the lowest-energy hole subband. We thereby deduce the parameter $\sigma_{0}=(\hbar / m \Omega)^{1 / 2}$ to characterize the spatial extent of $\varphi_{1}(z)$ at $B_{\|}=0$. The effect of $B_{\|}$is to confine further the hole wave function according to the relation

$$
\sigma_{B}^{2}=\sigma_{0}^{2} /\left(1+\sigma_{0}^{4} \ell_{B}^{-4} / 2\right),
$$



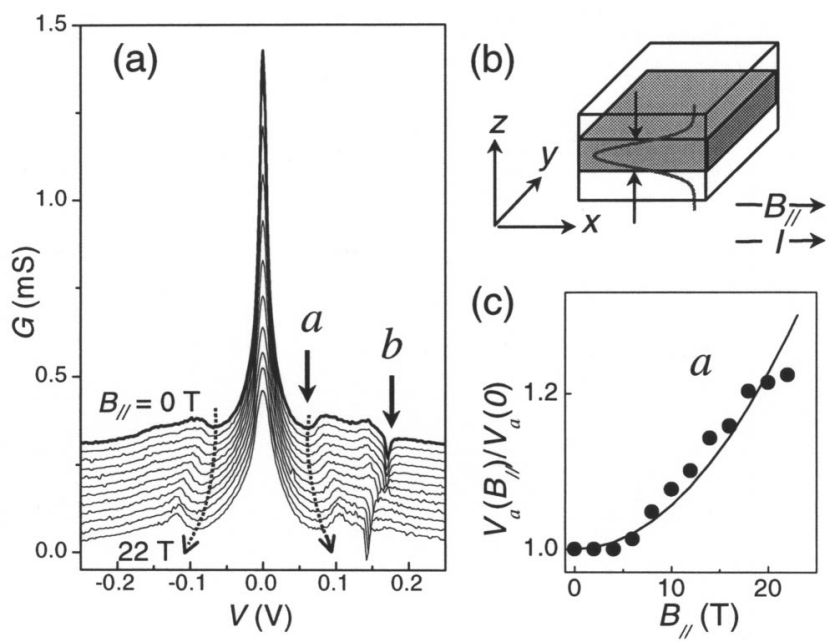

FIG. 3. (a) $B_{\|}$dependence of $G(V)$ at $4.2 \mathrm{~K}$. The magnetic field is parallel to the current direction and varies in $2 \mathrm{~T}$ steps from 0 to $22 \mathrm{~T}$. For clarity, the $G(V)$ plots are offset along the vertical axis. (b) Sketch of the geometry of the magneto-transport experiment. (c) $B_{\|}$dependence of the voltage position $V_{a}$ of feature $a$. The continuous line is a fit to the data by a parabolic model of the magnetoconfinement.

The additional confinement provided by $B_{\|}$tends to shift the inflection point $k_{m}$ to higher values of $k\left(k_{m} \sim 1 / \sigma_{B}\right)$. This implies that with increasing $B_{\|}$, larger biases, $V\left(B_{\|}\right)$ $\sim k_{m}^{2} \sim \sigma_{B}^{-2}$, are required to reach feature $a$ in the $I(V)$. In Fig. 3(c), we plot the observed voltage position $V_{a}$ of feature $a$ as a function of $B_{\|}$. It can be seen that $V_{a}$ increases approximately quadratically with $B_{\|}$and is well described by the relation $V_{a}\left(B_{\|}\right) / V_{a}(0)=\sigma_{0}^{2} / \sigma_{B}^{2}=1+\sigma_{0}^{4} \ell_{B}^{-4} / 2$, where $\sigma_{0}$ is a fitting parameter equal to $\approx 5 \mathrm{~nm}$. Our data deviate from the theoretical curve for $B_{\|}>18 \mathrm{~T}$. For these high magnetic fields, the magnetic length $\ell_{B}=6 \mathrm{~nm}$ becomes comparable to the size of the hole wave function, a regime in which our perturbation theory model cannot be used. The value of $\sigma_{0}$ is close to that $(\approx 4 \mathrm{~nm})$ obtained by numerical calculation of the Schrödinger equation for a $15 \mathrm{~nm}$ wide QW at $B_{\|}=0$. This result strongly supports our assertion that feature $a$ is related to the inflection point $\alpha$ in the HH1 subband. Thus, the shift of $a$ to higher bias with increasing $B_{\|}$results from the shift of $\alpha$ to higher $k$, due to the compression of the wave function.

Finally, we consider the origin of the narrow, but distinct, region of negative differential conductance (NDC) labeled $b$ in the $I(V)$ curve shown in Fig. 2(a). It occurs at $V_{b} \approx 180 \mathrm{mV}$. Note that the measured $B_{\|}$dependence of $b$ differs substantially from that of $a$ [see Fig. 3(a)], suggesting that the two features have a different physical origin. As can be seen from the energy dispersion curves in Fig. 2(b), at point $\beta$ states in the HH1 subband become degenerate with the broad minimum of the upper LH1 subband. This occurs at around $k_{[2}{ }_{23} 3 \approx 3.5 \times 10^{8} \mathrm{~m}^{-1}$. We tentatively propose that the origin of the NDC region is the acceleration of holes up to point $\beta$ in the dispersion curve, where they undergo scattering into the LH1 subband, thus significantly reducing their average group velocity. This process is analogous to the mechanism that gives rise to the Gunn effect in bulk $n$-doped GaAs at high electric fields and would partly account for the saturation of current at $V>V_{b}$. Further experiments and development of a model to include the effect of $B_{\|}$on the QW states and intersubband scattering rate are underway to determine the origin of feature $b$.

In conclusion, we have studied hole transport under the action of the intense electric field in a submicron planar p-type GaAs/(AlGa)As QW channel. We have shown that holes can be accelerated up to and beyond the inflection point in the highly nonparabolic lowest-energy subband of the QW. This leads to current saturation and negative differential conduction effects in the $I(V)$ characteristics. Finally, we have used a magnetic field to provide an additional confinement in the QW, thus mimicking in a given device the effect of decreasing the well width on the dispersion of the hole subband and on the non-linear transport properties.

This work is supported by the Engineering and Physical Sciences Research Council (United Kingdom).

${ }^{1}$ G. Bastard, Wave Mechanics Applied to Semiconductor Heterostructures (Les Editions de Physique, Paris, 1988).

${ }^{2}$ R. K. Hayden, D. K. Maude, L. Eaves, E. C. Valadares, M. Henini, F. W. Sheard, O. H. Hughes, J. C. Portal, and L. Cury, Phys. Rev. Lett. 66, 1749 (1991).

${ }^{3}$ L. Esaki and R. Tsu, IBM J. Res. Dev. 14, 61 (1970).

${ }^{4}$ R. R. Bashirov, Z. S. Gribnikov, N. Z. Vagidov, and V. V. Mitin, Appl. Phys. Lett. 77, 3785 (2000).

${ }^{5}$ C. D. Bezant, J. M. Chamberlain, H. P. M. Pellemans, B. N. Mudrin, W. Batty, and M. Henini, Semicond. Sci. Technol. 14, L25 (1999).

${ }^{6}$ A. J. Daneshvar, C. J. B. Ford, A. R. Hamilton, M. Y. Simmons, M. Pepper, and D. A. Ritchie, Phys. Rev. B 55, R13409 (1997).

${ }^{7}$ J. M. Luttinger and W. Kohn, Phys. Rev. 97, 869 (1955).

${ }^{8}$ P. N. Stavrinou and R. van Dalen, Phys. Rev. B 55, 15456 (1997).

${ }^{9} \mathrm{We}$ assume that the sizes of the channel are limited by the thickness $l_{d}$ $\approx 0.1 \mu \mathrm{m}$ of the depletion regions that form on each side of the constriction. The effective width, $w_{\text {eff }}$, is given by $w-2 l_{d} \approx 0.3 \mu \mathrm{m}$, where $w$ is the nominal width of the constriction $(w=0.5 \mu \mathrm{m})$. The effective length, $l_{\text {eff }}$, is equal to $2 \tilde{l}$, where $\widetilde{l}$ is the distance from the centre of the constriction at which the channel width becomes equal to $2 w_{\text {eff }}$. By geometrical considerations, we derive that $\widetilde{l} \approx l_{d}$ and $l_{\text {eff }} \approx 0.2 \mu \mathrm{m}$.

${ }^{10}$ M. Heiblum, K. Seo, H. P. Meier, and T. W. Hickmott, Phys. Rev. Lett. 60, 828 (1988).

${ }^{11}$ D. Sprinzak, M. Heiblum, Y. Levinson, and H. Shtrikman, Phys. Rev. B 55, R10185 (1997).

${ }^{12}$ J. Shah, A. Pinczuk, A. C. Gossard, and W. Wiegmann, Phys. Rev. Lett. 54, 2045 (1985).

${ }^{13}$ T. Elsaesser, J. Shah, L. Rota, and P. Lugli, Phys. Rev. Lett. 66, 1757 (1991). 